\title{
Using Solar Units for Wireless Sensor Network Nodes Deployed at Remote Areas: Border Monitoring Application Example
}

\author{
Ali Elkateeb \\ Associate Professor, University of Michigan, Dearborn, Michigan USA
}

\begin{abstract}
Wireless Sensor Networks (WSNs) are used in variety of applications. The amount of power consumed to perform the processing and communications by each node of WSNs will be related to the amount of communications between nodes, the type of applications, the type of processing core used in each node, and the type of components and technology used in the design of each node. One of the challenges associate with using such nodes in remote area is the power supplied to nodes of the WSNs. Although, using batteries could help to support the power of the sensor nodes used for some basic applications and for short period of time. However, using WSN nodes used in some applications such as border monitoring, cannot use batteries and continuous power supply to support such nodes with their working environment. In this research, we investigate this challenge for boarder monitoring application when the WSN nodes used in remote areas where it is not possible to use continuous power supply and batteries for powering such nodes.
\end{abstract}

Keywords: Wireless Sensor Networks (WSNs), Solar Units, Remote Areas, Border Monitoring Application.

\section{INTRODUCTION}

The WSNs offer economically viable solutions for applications that can be found in different settings such as industry, military, environment, health, etc. [1 - 4]. Border monitoring application has recently become an important application for many countries around the world. Instead of building expensive walls, the use of WSNs could provide a cost effective border monitoring system with less cost.

Typically, Nodes of WSNs are very small and integrated sensing, computing, communication units, and some other application-dependent units. Sensor nodes are designed using low-power embedded processor and other components that integrated on a small nodes' Printed Circuit Board (PCB). These nodes are programmed and designed to match the needs of the target applications. They are using low-cost and low-power components.

Using nodes for border monitoring applications to detect unlawful activities such as crossing the border by intruders will require the use of the vision sensor nodes. These nodes are required to continuously operate to monitoring the boarder and perform image processing to detect the intruders type. Therefore the vision sensor nodes will conduct more processing than that performed by some other WSN nodes used to serve other applications where sensor node is used to monitor temperature or humidity, etc. Performing sensor nodes for border monitoring applications will consume larger power than nodes used in other simple applications. Moreover, sensor nodes used in such simple applications will not require the use of fast and high performance processing units. Present nodes used in many WSNs applications are using low-performance embedded processor. Using WSNs in border monitoring applications will require the use of processing unit that can perform image processing and other sensor node function fast. Such nodes will require using processing cores provides good performance. In addition, the processing core will need to perform other node's processing functions that could be unknown at the early stage of node design until the application type is fully defined. Therefore, the use of the Field Programmable Gate Array (FPGA)-based processing core will be good approach to be used in WSN nodes design. Unfortunately, the FPGA is a power hungry technology and consume more power than other nodes implemented with other processing technology. Moreover, design challenge with using nodes located in remote areas is providing power to nodes located in remote area. In addition, using batteries for WSNs nodes are not possible due to the fact that such batteries will run out of power after short time and replacing these batteries for large number of WSN nodes will be costly and not practical for nodes distributed over large area.

For border monitoring application, the WSNs nodes should contain components required for such application such as Pixels sensors that will perform the function of a camera that will check the border for intruders. Also, small display unit can display the captured image of the intruder, which will work to monitor the border and check the type of the captured intruder image. In addition to these components, the sensor nodes will require high performance processing unit, memory, communication unit, and other components required for the sensor nodes operation.

In this paper, we present the vision sensor node (VSN) operation and design. Vision sensor node components and structure has been discussed along with the use of solar panel to provide power to VSN. 


\section{SENSOR NODE OPERATION FOR BORDER CONTROL APPLICATION}

As many countries are currently trying to protect their borders and looking for possible approach to achieve that, using sensor networks can be considered as one possible and cost effective solution. To control border invasion by intruders, a motion sensor can be used to detect any activates on the border. Despite the fact that using such approach will be a cost effective approach, however, such approach will not provide a good solution to this problem. Using motion sensors will no able to distinguish between human and other motion objects. Therefore, the most effective type of nodes that can be used for this application is the use of vision sensors, where the type of object can be identified and reported to the border authorities. However, the vision sensor node will need to continuously monitor the border and report the monitoring result to the border authorities. Therefore, using vision sensors can be more appropriate for detecting the motion objects and identifying the type of that object. The structure of the vision sensor will use the pixels sensor; processor that process the capture image to recognize the type of image; and a transmitter to transmit the captured image to the base station for image identifications. To perform all these activities of the vision sensors will consume large amount of power of the node. Supplying power to vision sensor nodes of the WSN in remote area, will be one of the main challenging problems that will be addressed in this paper. Other challenges with the use of sensor nodes can be located on supporting remote hardware modification/reconfigurations andw remote software reprogramming capability that can be very useful for border monitoring application. However, this capability is not addressed in this research and addressed in our other research $[5,7,11,12,13]$. Providing sensor nodes infrastructure with remote hardware modifications capability will allow switching between motion and vision sensors dynamically to use the right sensor at the right time and according to the application needs. For example, as the motion sensor detect a motion object then the vision sensor will be triggered to work and switched from idle condition to working state. Such working conditions of sensors can safe node's power where the needed components of the node will be switched to working conditions when that component will be required to function. The remote hardware modification capability will be implemented in every processing node to reconfigure the node resources from a remote distance and according to the application needs. Clearly, the use of the remote hardware modifications capability in sensor node can be a useful design feature if it is provided with sensor nodes. The sensor node can then be capable to support remote software reprogramming capability in addition to remote hardware modifications and can adjust the node design requirement to match the application needs. Unfortunately, the present FPGA design tools are not supporting remote hardware modifications, but they provide support for hardware modification performed through using the JTAG interface provided with FPGA chips. Each sensor node will include two sensors, the vision sensor (use a CMOS pixels sensor) and a motion sensor. Providing sensor node with vision sensor could require keeping the node working continuously to detect intruders, which will drain the node batteries quickly. Therefore, node infrastructure will start operation by enabling the motion sensor detect intruders movements and when such movement detected, the node processing unit will activate the vision sensor to operate. Such node design will make the sensor node minimize the power consumption. Therefore, using the motion sensor at starting time will minimize nodes power consumption for border monitoring application. As the motion sensor cannot identify the type of the motion object, the motion sensor can provide a signal to the node's processing unit when a motion is detected to activate the vision sensor to capture the image of the intruder and analyze the type of the object whether it is human or animal or car or whatever that object will be.

Vision Sensor Node

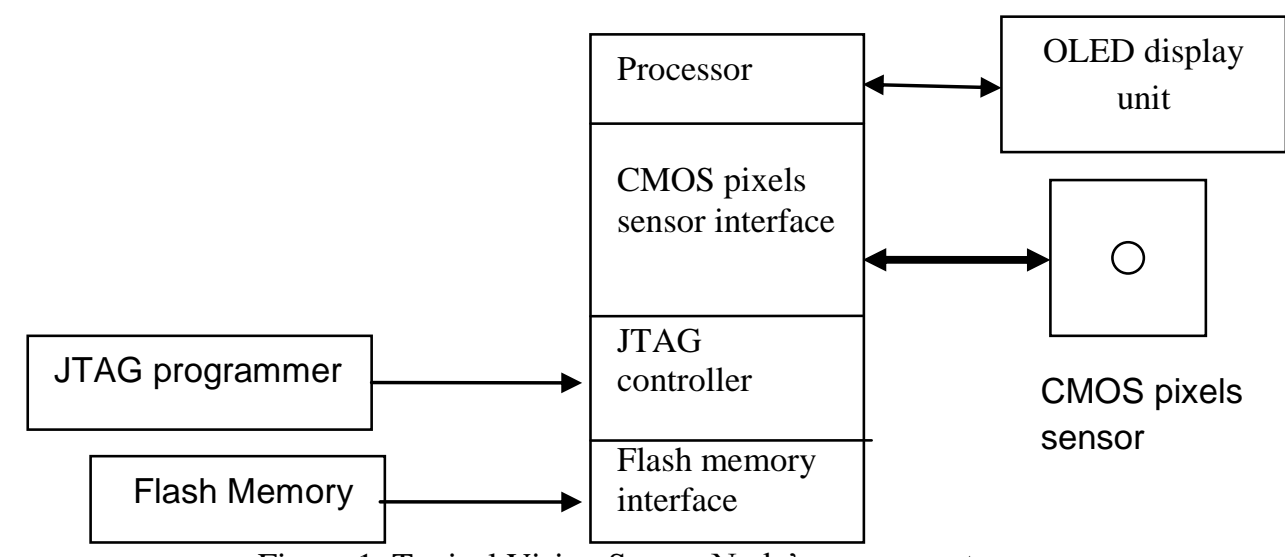

Figure 1 Typical Vision Sensor Node's components

Each vision sensor node contains the following components integrated on a small PCB of the node. Each vision sensor node will include the following components on the FPGA chip of the node: processor, sensors interfaces, small 
Memory, and JTAG controller. Other off-the-shelf components such as: CMOS pixels sensor and OLED display unit will be placed on the small PCB board ot the sensor node (Figure 1).

The processor for vision sensor node will be a custom designed soft-core type that optimized for border monitoring application. The processor has only the components required for this application in order to minimize node's power consumptions. The existing WSNs nodes are generally using standard processor core for their processing need. The ATmega128 micro-controller can be considered the most commonly used processing element for sensing nodes. However, the ATmega128 is a general purpose architecture, which is not optimized for border monitoring application. Therefore, it will consume more power than a specially designed processor. Some soft-core processors are currently available such as Microblaze, ARM Cortex, etc. Using such cores in sensor nodes can be useful to use with other components to implement a sensor node quickly. However, using these processing cores has a drawback in which they have many resources that may not be required to use in target application and, therefore, they will consume more power. Design special soft-core processor architecture for sensor nodes will be customized nodes design to be optimized, flexible, and efficient for the target WSNs applications use.

We have used non-pipeline MIPS architecture for the soft-core processor since the MIPS core is simple and efficient architecture. Also, the processing core is small and can be integrated on the low-power FPGA chip. A specialized 32bit non-pipelined MIPS soft-core processor has been developed for the sensor node design [Figure 2]. The developed soft-core processor has a performance of 10 million instructions per second. Such performance is acceptable for many sensor Network applications $[8,9,10]$.

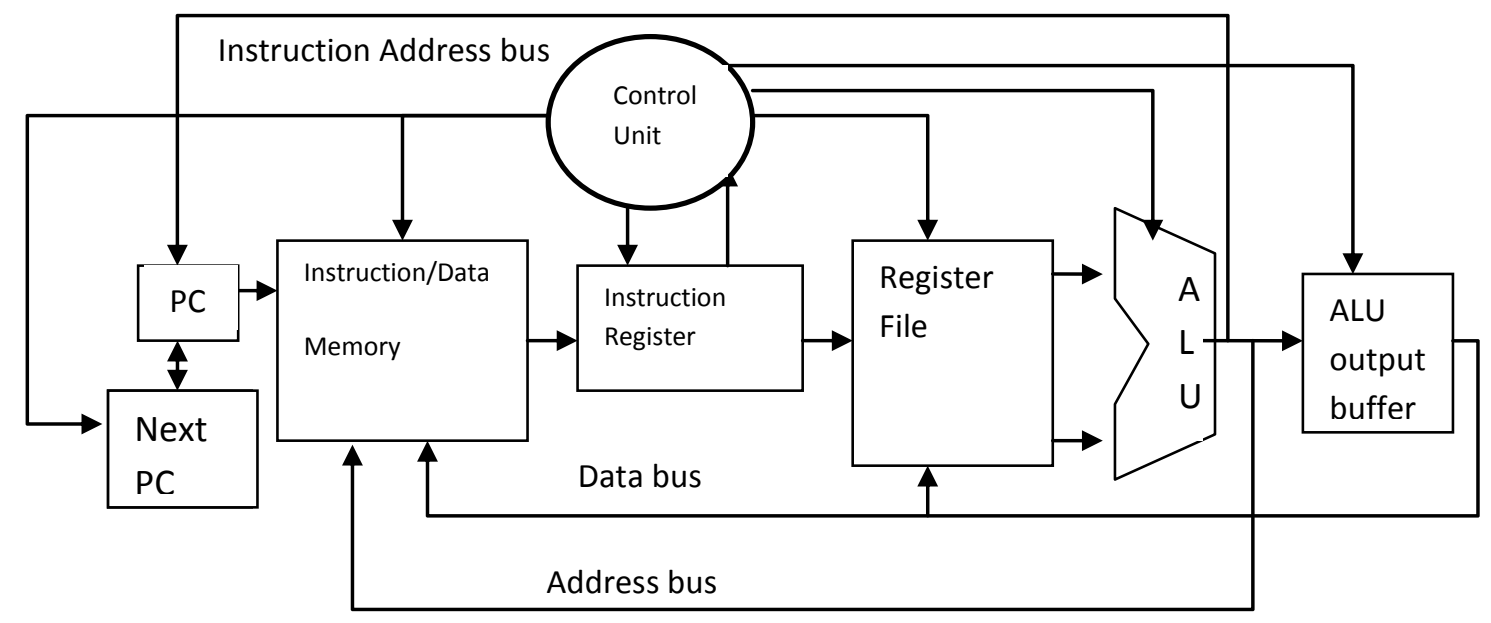

Figure 2: Soft-core processor architecture developed for RH-node

The organization of the node's components of the base station is as shown in Figure 3.

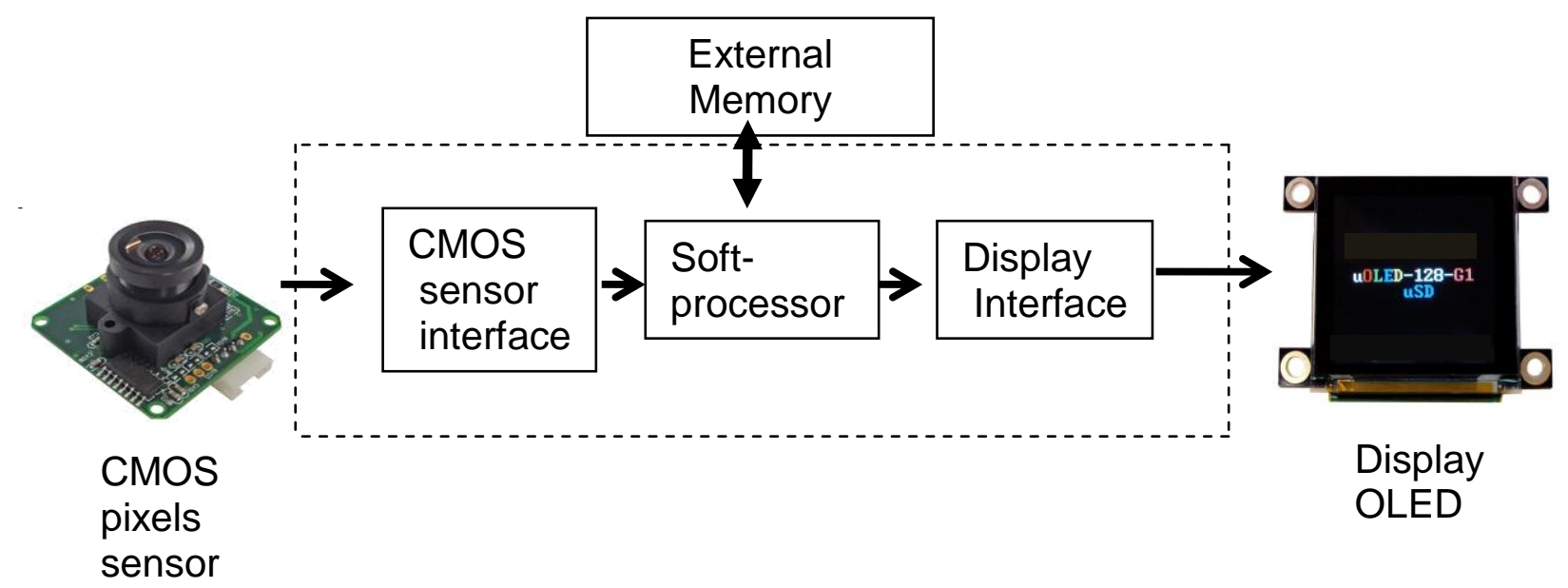

Figure 3 Typical structure of the Base station sensor node 


\section{SOLAR UNIT AS A SOURCE OF POWER FOR SENSOR NODES}

One of the main issues for the border monitoring is the use of sensors that will be deployed in remote areas. Typically, sensors nodes could work for a year using AA batteries. However, for border monitoring applications, sensors use FPGA technology, perform extensive image processing, and therefore, nodes will consume all their energy at short time. The use of FPGA chip at each node as the processing core for each sensor node will consume large amount of power. The power of the sensors can be reduced by using some of the recently developed low-power FPGA chips. This may include Actel Igloo, Xilinx Spartan 6, etc. Moreover, to cope with sensors power consumptions, a solar-based unit used in sensor node will include the solar panel and the interface for such a unit can be used to deliver power to each sensor node. As solar panels are currently available in small sizes and are relatively inexpensive, they become feasible and acceptable to be used for recharging the batteries of the sensors to provide them with longer working time even when the FPGA is used in the sensors design.

It is clear that the sensors will have difficulties to sustain their power in some remote locations, where sunlight intensity and duration of light availability is relatively low, such as in arctic region. However, sensors that use solar panels can still be efficiently used in some applications such as border monitoring, where sensors are used in areas that have the sunlight available for long periods of time. We could design a solar charging unit for the sensors using the solar panel as the one shown in Figure 3. However, there is no currently available off-the-shelf FPGA chip that has a power provided to the FPGA chip from solar panel.

\section{SOLAR POWER PROVIDE TO EACH SENSOR}

Typically, using a solar panel to power each sensor node will be affected by the existing Sunlight. Sensor panel shown in Figure 4 present one type of solar panel that can be used in every sensor node to provide power to every node. These panels are small and inexpensive. The panel will operate during the day and night times to provide the required power for each sensor node Figure 5. We evaluated the amount of the power consumed by the processing unit of the sensor node using Xilinx FPGA Spartan-6 chip. This evaluation was performed using by a Xilinx XPower Analyzer package. The power consumption of the node has a processing core support 12 instructions and a register file of 32 registers, 32 bits each. The FPGA-based processing core will require $38.8 \mathrm{mAh}$ to evaluate the processing of an intruder movement.

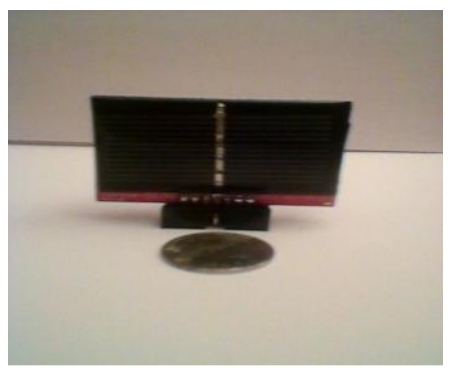

Figure 4 Tiny and inexpensive solar panel unit

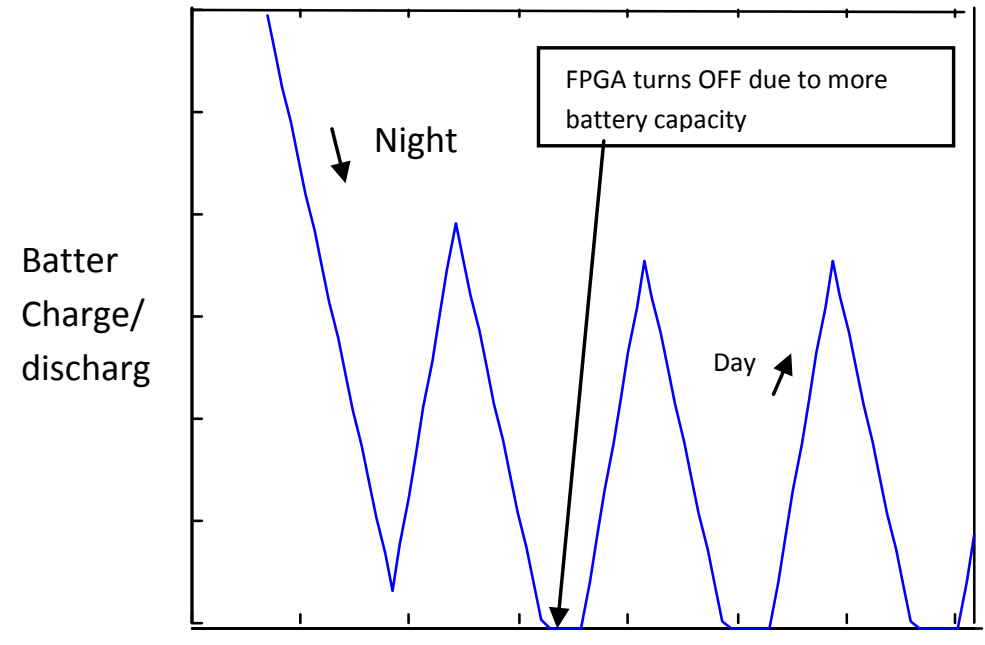

Figure 5

FPGA shuts off time for solar hours/day

Table 1: Number of working hours before vision sensor node will run out of power

\begin{tabular}{|l|l|}
\hline \multicolumn{1}{|c|}{ Solar Hours per Day } & Number of Hours when the FPGA Shuts Off \\
\hline 2 & 318 \\
\hline 3 & 602 \\
\hline 4 & 812 \\
\hline 5 & 1003 \\
\hline
\end{tabular}


One way to gauge the ability for the solar unit to power the FPGA is to understand the time until the FPGA consumes all of its power and shuts off. This time corresponds to 0 battery capacity when there is no solar power generated and saved in the rechargeable batteries of the sensor's processing unit. As the solar panel, shown in Figure 4, uses 2.4VDC and can generate $80 \mathrm{~mA}$ at direct solar incidence, the time when the Spartan-6 chip will shut off and runs out of power is shown in table 1. We have fixed the Solar Hours per Day on each row of the Table 1 shows the duration when the FPGA chip loses the power and shuts off. The simulation results show that the nodes will able to continue working for long time and may shut off under the situation when the sunlight is not available for certain period of time, which may unlikely happen. However, more research work will be performed in this project to evaluate sensors running out of power in response to the existing day sunlight.

\section{CONCLUSION}

The vision sensor node can be used for border monitoring applications. In this paper, we presenting the design of such node using CMOS pixels sensor, custom made processor, solar panel, and other components. Using solar panel components to provide power to vision sensor nodes can be an effective approach when such nodes work at remote area. The use of solar panel can support the vision sensor nodes with power for long period of time even when these nodes use an FPGA processing units that consume large power.

\section{REFERENCES}

[1] G. J. Pottie and W. J. Kaiser, “Wireless Integrated Network Sensors,” Commun. ACM, vol. 43, no. 5, May 2000, pp. 551-558.

[2] J. M. Kahn, R. H. Katz, and K. S. Pister, "Next Century Challenges: Mobile Networking for Smart Dust," Proc. ACM MobiCom'99, Washinton, DC, 1999, pp. 271-278.

[3] I. Akyildiz, W. Su, Y. Sankarasubramaniam, and E. Cayirci, “A Survey on Sensor Networks,” IEEE Communications Magazine, pp. 102-114.

[4] D. Culler, D. Estrin, and M. Srivastava, "Overview of Sensor Networks", IEEE Computer, August 2004, pp.41-49.

[5] Ali El Kateeb, "Hardware Reconfiguration Capability for Third-Generation Sensor Nodes", IEEE Computer, May 2009, pp. 95-97.

[6] Ali EL Kateeb and Lubna Azzawi, "Hardware Reconfiguration Capability for Third Generation Sensor Nodes: Design and Challenges", the $22^{\text {nd }}$ IEEE International Conference on Advanced Information Networking and Applications, March 2008, Japan, pp675-680.

[7] A. Elkateeb, "Supporting Next-generation Wireless Sensor Network Nodes with Remote Hardware Modification Capability", Proceedings of IEEE int'l Conference on Wireless for Space and Extreme Environments, November 2013, Baltimore, USA.

[8] Ali El Kateeb, R. Aiyappa, and L. Alazzawi, "Sensor network processor", Canadian electrical and Computer Engineering Conference, May 2008, pp1031-1034.

[9] Ali El Kateeb, Aiyappa Ramesh, and Lubna Azzawi, “ Wireless Sensor Nodes Processor Architecture and Design”, the 22nd IEEE International Conference on Advanced Information Networking and Applications, March 2008, Japan, pp892-897.

[10] R. Aiyappa, Sensor Networks Processor, Master thesis, University of Michigan-Dearborn, January 2010.

[11] Ali EL Kateeb and Lubna Azzawi, "Hardware Reconfiguration Capability for Third Generation Sensor Nodes: Design and Challenges", the 22nd IEEE International Conference on Advanced Information Networking and Applications, March 2008, Japan, pp675-680.

[12] A. El Kateeb," RH-mote for Next-generation Wireless Sensor Networks", 4th International Conference on Emerging Ubiquitous Systems and Pervasive Networks (EUSPN-2013), Ontario, Canada.

[13] Ali EL Kateeb and Lubna Azzawi, " Hardware Reconfiguration Capability for Third Generation Sensor Nodes: Design and Challenges", the $22^{\text {nd }}$ IEEE International Conference on Advanced Information Networking and Applications, March 2008, Japan, pp675-680. 